SII-2b

\title{
Comparable numerical study on different working fluids for micro scale power cycle integrated into a pellet fired boiler
}

\section{Numerical Simulation of the Tesla Turbine Efficiency}

\author{
Roberto Lisker ${ }^{1}$; Mario Nowitzki²; Udo Hellwig³; Franz Xaver Wildenauer ${ }^{4}$
}

\begin{abstract}
A cost-effective power cycle shall be developed to supply heat and electricity (co-generation). The aim of this study is to find an appropriate power cycle and working fluid for a friction turbine application. Due to its low production costs, better scalability and low-maintenance requirements, a friction turbine, also known as Tesla turbine, shall be used. In order to find a proper working fluid and estimate the efficiency and power output, a numerical study was performed on five promising working fluids namely Xenon, Argon, Helium, Air and superheated Steam. The simulation model consists of three disks with a diameter of $0,25 \mathrm{~m}$ and an outlet diameter of $0,12 \mathrm{~mm}$ rotating at constant $10.000 \mathrm{rpm}$. It is tried to keep the Gap Reynolds number at a constant 73, to ensure a laminar flow regime between the disks. The outcome of the numerical study showed that all working fluids are suitable. The highest efficiency (shaft power / technical power) has been achieved with steam (96\%) and a power output of $126 \mathrm{~W} / g a p$. The least efficiency with $89 \%$ has been realized with air and a Power output of $118 \mathrm{~W} / g a p$. The noble gases are in between these fluids.

By comparing the simulation data and taken into account numerical and estimation errors, all five working fluids are at par. Thus, the choice of the working fluid and the power cycle is an economical. While a hot gas air turbine is not as efficient as the steam turbine, the invest and maintenance costs are lower due to less heat exchangers and necessaries like water reconditioning and a phase separator.
\end{abstract}

\section{Introduction}

For domestic heating, biomass is seen as an adequate substitution of fossil fuels like oil or natural gas. Combusting biomass (here pellets) is recognized as non-climate hazardous since the amount of Carbon dioxide emitted is equal to the one absorbed (climate-neutral). However, for domestic applications, solid biomass-based co-generation units which produce heat and electricity are not commercially available yet. The main cause is fouling and slagging of the convective and radiative heat exchanging surfaces due to the high amount of ash residues in the flue gas, making it impossible for the direct use in a gas-turbine or gas-engine. To overcome this issue and to establish a power cycle, the combustion process must be separated from the power

\footnotetext{
${ }^{1}$ Dr. Roberto Lisker, Technical University of Applied Sciences Wildau, Wildau

2 Dipl.- Ing. Mario Nowitzki, Technical University of Applied Sciences Wildau, Wildau

${ }^{3}$ Prof. Dr.-Ing. Udo Hellwig, ERK Eckrohrkessel GmbH, Berlin

${ }^{4}$ Prof. Dr. rer.nat. Franz Xaver Wildenauer, Technical University of Applied Sciences Wildau, Wildau
} 
cycle. Thus, an external combustion chamber is used to transfer the heat of combustion to a clean working fluid which drives the turbine generator set.

For the cycle design a commercially available pellet fired boiler (Ökofen PES 56) has been selected which shall be equipped with a micro scale power cycle. The pellet fired boiler has a nominal thermal output of $56 \mathrm{~kW}_{\text {th }}$ and the boiler efficiency is at $93 \%$. The maximum flue gas temperature achieved in the furnace zone is $1400 \mathrm{~K}$ and the flue gas volume flow is $0,04 \mathrm{~m}^{3} \mathrm{~s}^{-1}$. [1]. The power unit consists of heat exchangers to transport the heat from the furnace to the power cycle and heating system, a turbo-set consisting of a friction turbine, a compressor or pump which circulates and pressurizes the working fluid and the generator which produces a nett power output of $3,5 \mathrm{~kW}_{\mathrm{el}}$.

At the moment, the system is in an early stage of development. For the power unit, two processes are conceivable. The first one is a classic Rankine Steam Cycle. Here the working fluid is steam who drives the turbine. The second one is an Ericsson process, a closed gas turbine process, where a gaseous working fluid drives the turbine.

In order to make a final decision about the cycle, the performance of the friction turbine and the turbine efficiency shall be evaluated regarding the working fluid. The working fluids to be evaluated are Steam, Helium, Argon, Xenon and Air. Therefore, parts of the turbine geometry have been modeled and numerous numerical simulations have been done to evaluate the turbine performance.

\section{The Power Unit}

The power unit shall be integrated in the pellet boiler, a basic scheme is displayed in the following figure. Inside the furnace the heat exchangers are placed. The furnace wall will be used to recuperate the heat coming from the turbine and preheat the working fluid. The second heat exchanger is directly placed inside the furnace and heats the working fluid to the final temperature of $1273 \mathrm{~K}$. The rest of the heat is used to generate warm water or heating. The Turbo-Set shall generate a net output of $3,5 \mathrm{~kW}$ el under nominal conditions. Furthermore, it shall produce the energy necessary for the compressor and other electrical components of the boiler system (PLC etc.).

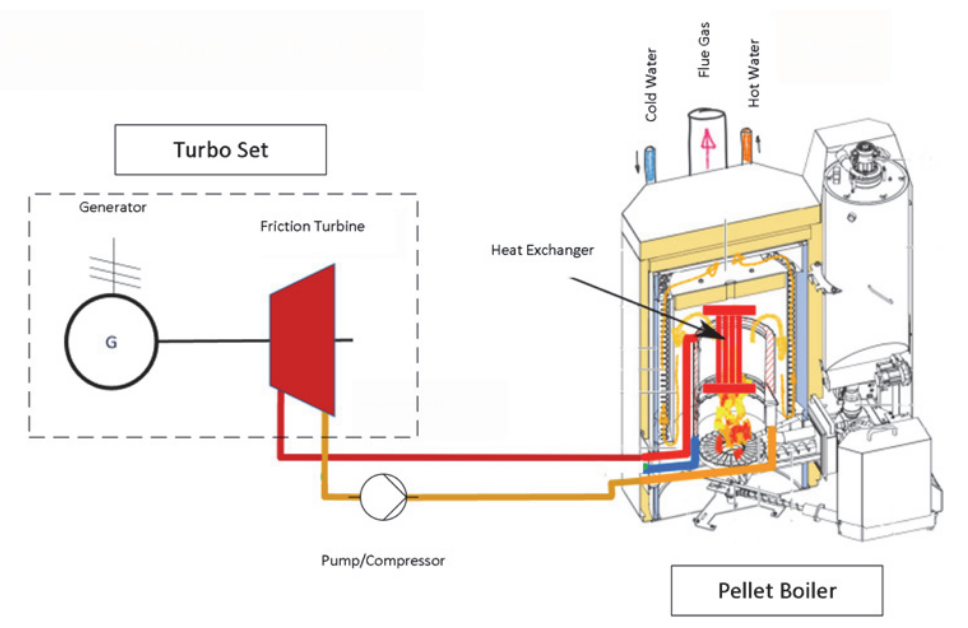

Figure 1 Basic elements of the Power Unit integrated into the pellet fired Boiler. On the left-hand side, the Turbo Set is displayed which is connected to the pellet fired boiler. 


\subsection{Ericsson Cycle}

The Ericsson Process is a reference cycle for gas turbines [2]. At first, the pressurized working fluid is heated to the desired turbine inlet temperature (4-1, Figure 2,left). The pressure is kept constant. Afterwards the Fluid is isothermal expanded (1-2, Figure 2, left) until it reaches the low pressure p', it leaves the turbine and enters the regenerator (2-3, Figure 2, left), who preheats the cold working fluid. Afterwards, the cold working fluid is compressed until the desired highpressure $p$ (3-4, Figure 2, left). The downside of the cycle is the compression of the gaseous working fluid. Due to the low density of the gaseous fluids the compression takes a lot of energy. In Figure 2 right, the area under the blue curve displays the specific labor needed to compress air at ambient conditions up to 10,5 bars. One can see, that in the range of 1 to 4 bars the majority of the compression energy is needed (Figure 2, red area). To overcome this, the cycle can be operated under pressurized conditions, whereas the lowest pressure $p$ ' is higher than the ambient pressure. The higher density accounts for significant energy savings.
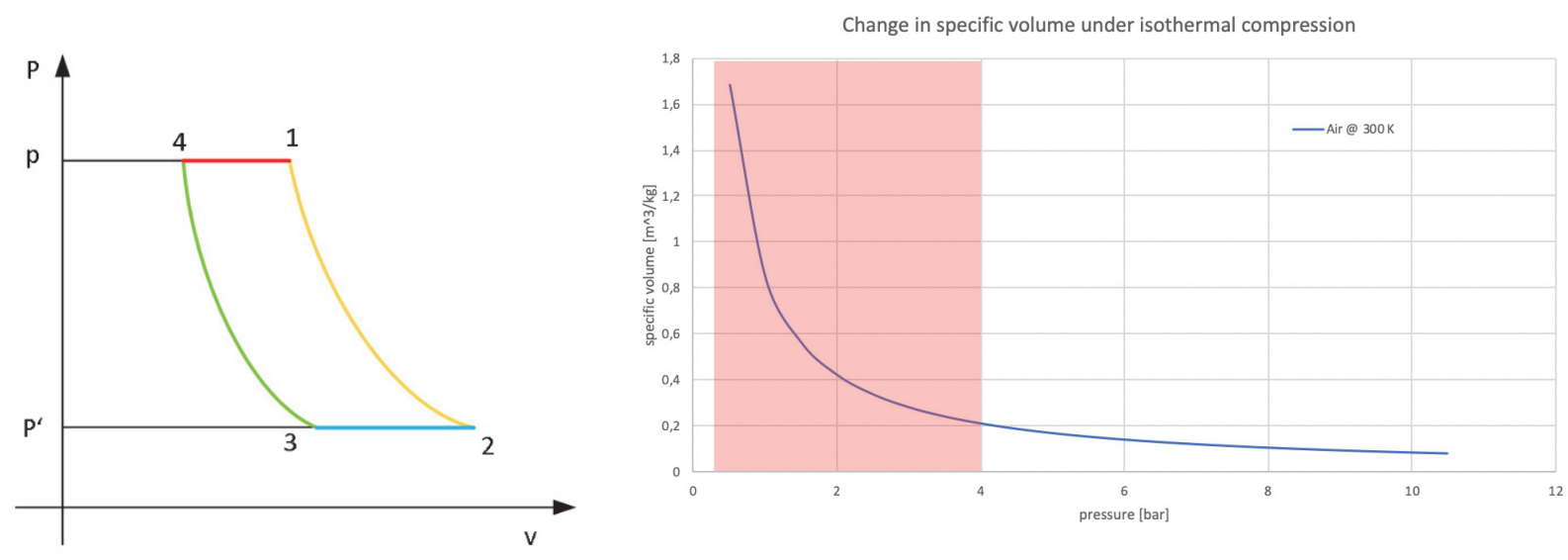

Figure 2 Left: Pressure-Volume Diagram of an Ericsson Process. Right: the change in specific volume of air while compressed.

In order to estimate the turbine size, the cycle has been roughly designed with air. In this case, the lowest pressure will be 4 bars and the highest pressure 9 bars. The turbine inlet temperature is set to $1173 \mathrm{~K}$ and the efficiency 0,6 . Like the turbine the compressor efficiency is estimated with 0,6 and it has to produce a volume flow of $0,0115 \mathrm{~m} 3 \mathrm{~s}-1$. From these calculations, the gross electrical power production of the turbo-set must be $11 \mathrm{kWel}$.

\subsection{Friction Turbine}

The friction turbine also known as Tesla Turbine, was invented by Nikola Tesla in 1909 [3]. It consists of several plane parallel discs arranged on a shaft. The distance between the discs is determined by the viscosity and the boundary layer thickness inside the gap. The working fluid enters the gap at the outer most perimeter and moves on a spiraling path towards the center of the disk, where the outlet is situated. In figure 3 left, a sectional view of a friction turbine model is shown. Due to the reduction in the cross- sectional flow area, the velocities are rising towards the outlet (see figure 3, right). Especially the tangential velocity accounts for energetic losses, which can be minimized by adjusting the gap width and/or rotation rate. In figure 3 the red dashed line represents the ideal tangential velocity. It should be just over the disk velocity, enough to produce the needed drag and minimize the energetic losses. 
The advantage of the friction turbine is its simplicity and robustness. Due to the parallel flow of the working fluid to the disk surface, the turbine is impervious against particle laden flows and condensate formation [4].
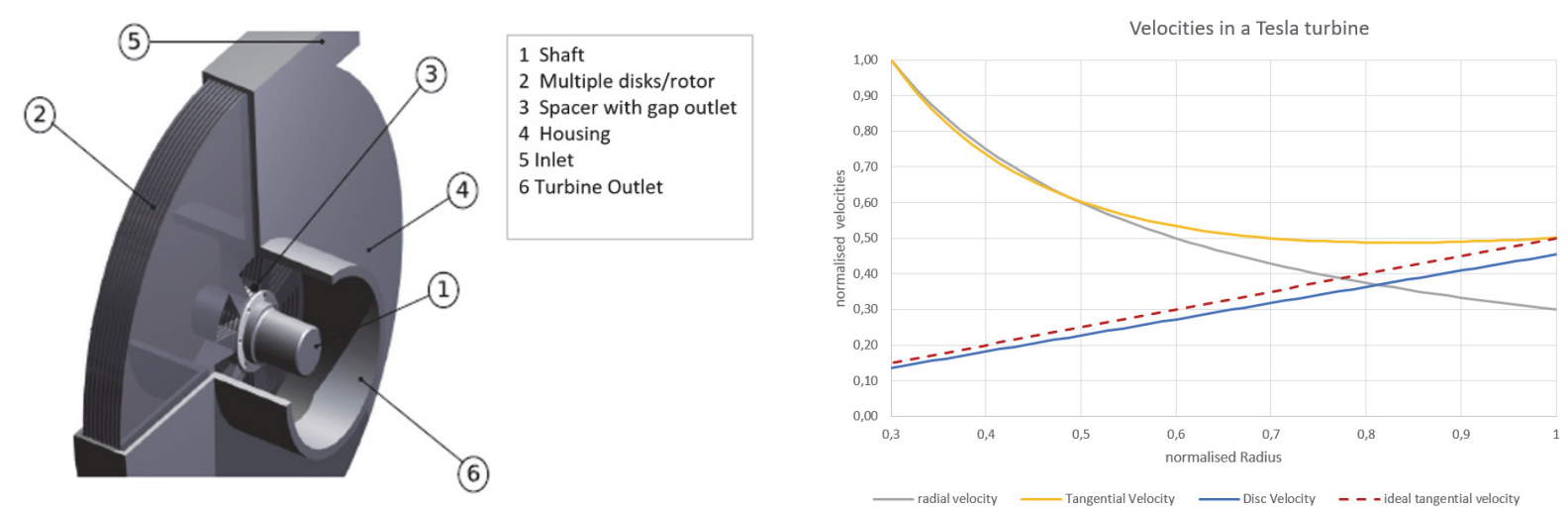

Figure 3 Left: Model of a friction turbine; Right: Normalized velocities inside a friction turbine gap element. The ideal tangential velocity is displayed as a dashed red line. The distance between the yellow and red line is the energetic loss

For the turbine assessment, the efficiency with respect to the working fluid and the gap Reynolds number are essential. The turbine efficiency is calculated according to the following equation

$$
\eta=\frac{\Delta h_{t o t}}{M \omega} \quad \text { eq. } 1
$$

whereas $\Delta h_{\text {tot }}$ is the total enthalpy difference between the in- and outlet of the turbine, $M$ the produced momentum and $\omega$ is the angular velocity of the flow. In order to make the simulations comparable, the gap Reynolds number $\left(R e_{\text {gap }}\right)$ will be used. It can be expressed with the inlet mass flow as follows:

$$
\operatorname{Re}_{\text {gap }}=\frac{{ }^{m} \phi}{2 \pi r \eta} \quad \text { eq. } 3
$$

whereas $r$ is the outer radius of the disc, ${ }^{m} \phi$ is the mass flow of the fluid and $\eta$ the dynamic viscosity. In order to obtain a high turbine efficiency, the gap Reynolds number should be kept low, which accounts for a laminar flow regime in the gap.

\section{The Numerical Model}

For the comparative simulation, a simulation model has been developed. It consists of a housing with two tangential inlets, three disks (two gap elements) and a reduced outlet in the center (see Figure 4 left). The original model consists only of one disk, but here the efficiency reducing effect of the gap formed between the housing wall and the rotor could not be modelled. Thus, a threedisk geometry is chosen resulting in 2 full fluid gaps and due to symmetry two half gaps on both sides. In the following table the geometric and operational data are displayed.

\begin{tabular}{|c|c|c|c|}
\hline Property & Value & Property & Value \\
\hline Disk outer radius & $0,25 \mathrm{~m}$ & Rotation rate & $10.000 \mathrm{rpm}$ \\
\hline Disk inner radius & $0,12 \mathrm{~m}$ & Inlet velocity & $140 \mathrm{~m} \mathrm{~s}^{-1}$ \\
\hline Gap width & $0,0003 \mathrm{~m}$ & Outlet pressure & $1 \mathrm{~atm}$ \\
\hline Gap Reynolds No. & 73 & Inlet temperature & $1273 \mathrm{~K}$ \\
\hline
\end{tabular}

Table 1 Geometric and operational properties of the simulation model 
Due to different fluid viscosities, the mass flow must be adjusted in order to maintain a constant gap Reynold number and inlet velocity. To set the mass flow, the inlet channels height is adjusted, in order to achieve a constant inlet velocity at a given channel width. Therefore, the housing inlet height is adjusted. Beforehand, a mesh study has been performed to optimize the calculation time and computational resources. The study has shown, -despite of the laminar flow between two discs-, that within the gap a large amount of mesh cells is needed in transverse flow direction. The reason being, the complex flow phenomena at the most outer and inner perimeter, which cause disturbances, and result into additional pressure and velocity fluctuations. Furthermore, the Mach number can locally exceed one, meaning supersonic flow. Thus, the stability of the numerical solver must be increased to handle local jumps in fluid properties (pressure surge). This can be done by a finer mesh in high Mach number areas. In total about 8 mesh cells are needed in transverse directions, of which 6 are prism layers. This yields to an overall mesh cell amount of about 30 mio. polyhedral cells for one gap element (see Figure 4).
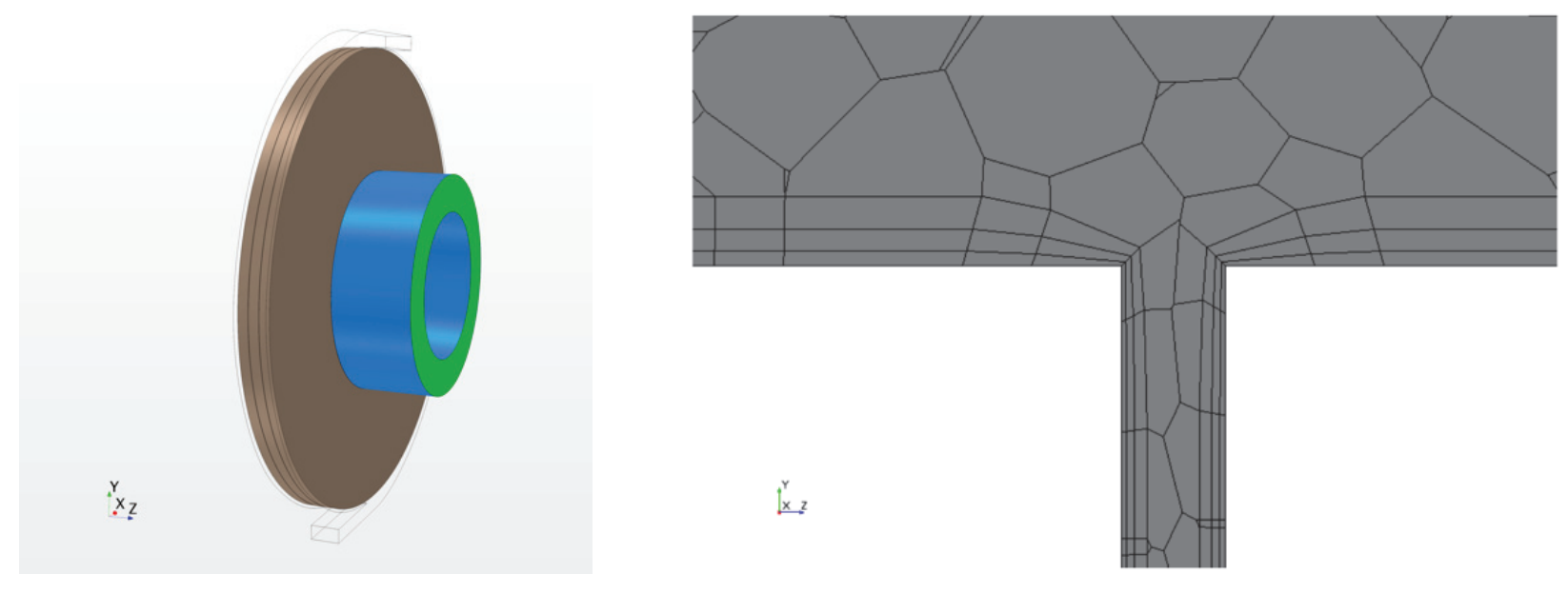

Figure 4 Left: The geometrical model of the turbine used for the simulation. The turbine consists of two inlets (displayed as wireframe) and an outlet in the center of the disks (blue- green). The disks are marked brown. Right: View of the polyhedral mesh at the outer most radius. One can see the prism layers close to the disk wall.

\subsection{Mathematical Model}

In order to compare different working fluids, the following settings have been done. The simulation is steady state, transient effects can be neglected, and a steady state model is used. An adiabatic expansion is assumed in the friction turbine, even though solving the energy equation is important to include enthalpy changes due to abrupt fluid acceleration/deceleration. One effect of abrupt enthalpy changes is the Joule-Thomson-Effect. Especially in the transition area from the gap to the hollow outflow shaft shall be mentioned. In order to account for this effect, a Real Gas model is needed. Thus, the Van der Waals model is chosen, demanding a laminar flow regime in the gaps. Even though a turbulence model is necessary for the simulation of the inlet housing and hollow outlet shaft. Thus, a transition model is needed which allows to switch between turbulent and laminar flow. Because of the high wall impact the SST Turbulence model is used. For the modeling of the change between turbulent and laminar flow, the Gamma Transition model applied. The models used in the simulation are shown in table 2. 


\begin{tabular}{|l|l|}
\hline Property & Model \\
\hline Time & Steady State \\
\hline Energy & Segregated Enbthalpy \\
\hline Turbulence & SST K $\omega$ \\
\hline Turbulence transition & Gamma transition \\
\hline Equation of state & Real Gas Van der Waals \\
\hline
\end{tabular}

Table 2 Used properties for the solver

\subsection{Boundary Conditions}

In this study the fluids have a constant inlet velocity of $140 \mathrm{~m} \mathrm{~s}^{\wedge}-1$ and an inlet temperature of $1273 \mathrm{~K}$. The hollow shaft outlet is a pressure outlet of $1 \mathrm{~atm}$. Onto rotating discs and inner shaft surface a wall tangential velocity of $10,000 \mathrm{rpm}$ is set to simulate rotation.

\section{Results}

In the table 3, the results of the simulations are displayed. One can see that the efficiency according to equation 1 is high. The highest efficiency has been achieved with Steam $(0,95)$ and the lowest with air $(0,89)$. As mentioned above the gap Reynolds number should be 73 for all simulations. To achieve the constant gap Reynolds number, the inlet housing height was adjusted with subsequently meshing. Due to the insufficient prediction accuracy of the pressure drop (sudden local fluid properties changes), the inlet height has been determined in an iterative process. Hence the gap Reynolds numbers is close to 73 the simulation results can be compared. An exception is air, here the local changes in fluid properties are so dominant, that a gap Reynolds number of 73 could not be reached. 82 was the closest achievable.

In the following table, the results of all simulations with corresponding fluids are displayed.

\begin{tabular}{|c|c|c|c|c|c|}
\hline Fluid & Argon & Air & Xenon & Steam & Helium \\
\hline Re gap & 73,91 & 82,48 & 73,76 & 74,56 & 73,28 \\
\hline velocity inlet $\left[\mathrm{m} \mathrm{s}^{-1}\right]$ & 140 & 140 & 140 & 140 & 140 \\
\hline inlet temperature [K] & 1273 & 1273 & 1273 & 1273 & 1273 \\
\hline outlet temperature [K] & 1269,49 & 1267,77 & 1277,67 & 1270,02 & 1270,53 \\
\hline technical power [W] & 92,71 & 131,91 & 117,63 & 133,6 & 151,44 \\
\hline shaft power [W] & 86,3 & 118,43 & 110,297 & 126,82 & 138,13 \\
\hline Efficiency [\%] & 93,09 & 89,77 & 93,77 & 94,92 & 91,21 \\
\hline Moment [Nm] & 0,082 & 0,113 & 0,105 & 0,121 & 0,132 \\
\hline Pressure drop [bar] & 0,253 & 0,328 & 0,534 & 0,413 & 1,213 \\
\hline Inlet height [mm] & 4,03 & 5,84 & 1,77 & 7,9 & 21,19 \\
\hline
\end{tabular}

Table 3 Summarized results of the numeric simulation

An interesting result is, that despite of different fluids with different properties, the turbine efficiency is close. That implies a dependency of the efficiency on the gap Reynolds number. By taking a look at the shaft power, there are noticeable differences between the fluids. Argon generates the lowest shaft power, although the efficiency is high. Helium has the best shaft power, but the efficiency is the second last. Here a correlation between the shaft power and the 
pressure drop matches better. If the pressure drop is high the shaft power is high too, except for Xenon. Xenon is a high-density gas, so the low shaft power and high pressure- drop could be caused by increased inertia forces. In general, a high shaft power is desirable. Helium, which achieved the highest shaft power of $138,1 \mathrm{~W}$, also has the highest pressure drop, more than double than of Xenon which has the second highest pressure drop. In comparison the power output is only $27 \mathrm{~W}$ lower. Another fluid worth mentioning is steam. Here the highest efficiency has been achieved and the shaft power with $126,8 \mathrm{~W}$ is the second highest. Yet the pressure drop is only a third of Helium.

In the following figure 5 left, the total velocity $\left(c=\sqrt[2]{c_{\text {tangetial }}^{2}+c_{\text {radial }}^{2}}\right)$ of the working fluids in the middle of the gap is plotted. By examine the velocities, the high pressure drop of Helium can be explained with Bernoulli's principle. An increase in velocity towards the outlet increases the pressure drop $\left(\frac{e c^{2}}{2}\right)$ consequently. Furthermore, for the evaluation, the disk velocity is plotted (red line). In order to obtain an efficient turbine, the tangential fluid velocity should be just over the disk velocity like Xenon or Air. In figure 5 right, the radial velocities are plotted. One can see, that Helium reaches by far the highest velocities at the turbine outlet. The reason is the low density of Helium, which requires a higher volume flow to achieve the same mass flow as the other fluids. This is congruent to the inlet height of the helium simulation. Here the inlet height is more than ten times bigger than Xenon and more than 2 times higher than steam (see table 3). Regarding the velocities, pressure drop, mass flow and shaft power, Steam, Xenon and Air seems to be promising candidates. In terms of efficiency, all fluids are at par.
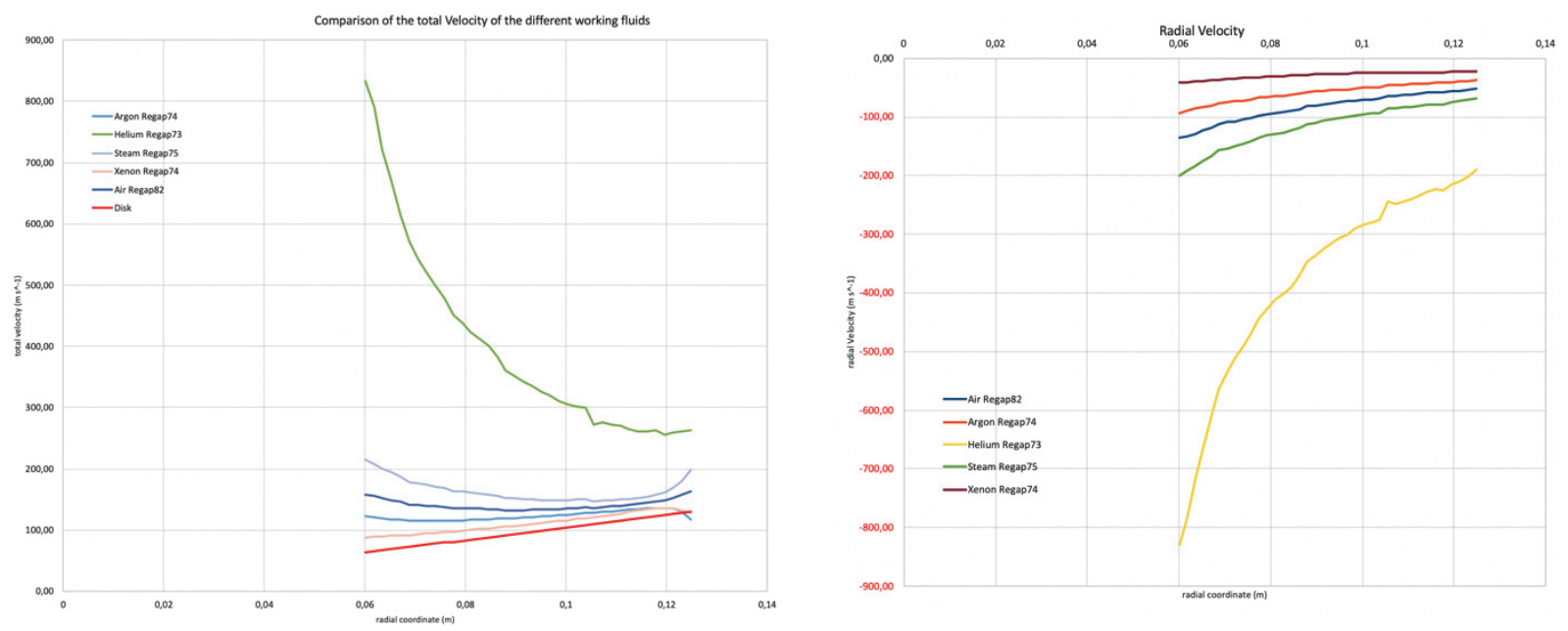

Figure 5 Left: Comparison of the total velocity of the working fluids in the gap the red line represents the disk velocity. For an ideal turbine, the velocities should be as close as possible to the disk. Right: Radial velocities in the turbine gap

\section{Conclusion}

The goal of this study was to find a proper working fluid for a pellet fired boiler power cycle. In terms of efficiency all working fluids are suitable. They can be regarded as equal. Even though, the efficiency is equal, the shaft power differs. But this can be adjusted by increasing or lowering the number of disks to reach the final necessary power output. Thus, the decision of the working fluid is based on economical reasons. Regarding the turbine, the best balance between shaft 
power, efficiency and pressure drop has superheated steam, followed by Xenon, Air and Argon. But the turbine is only one part of the cycle. First of all, the size of the compressor determines the additional power the generator has to deliver. A power cycle based on Helium would have a much higher compressor power consumption, which reduces the cycle efficiency. A Rankine Cycle would have the lowest power consumption due to the high density of water. The Ericsson Cycle with the lowest power consumption should be the Xenon followed by Argon and Air. Regarding the heat transfer, the picture is contrary. Helium has a higher thermal conductivity compared to the other working fluids, meaning the heat exchangers are smaller. Compared to the other working fluids, Xenon needs the biggest heat transfer surface, making it doubtful whether such a heat exchanger could be integrated in a pellet fired boiler. In comparison to Helium the heat exchanger surfaces need to be at least 25 times larger. The heat transfer surface regarding Steam and Air is only 5 times larger than Helium. Larger heat transfer areas account for higher invest costs so the system would be more. For the pellet boiler power unit, the Ericsson cycle is preferred over the Rankine cycle, due to the lower complexity. For domestic power cycles Air has shown the best balance between, pressure drop, shaft power and heat transfer sizes. Nevertheless, despite of the presumably higher power consumption due to the needed higher volume flow rate, Helium might be an interesting alternative because of the smaller necessary heat exchanger surface.

\section{Outlook}

With the gained results, first insights on the development process ahead are given. It can be concluded that the economical aspect cannot be neglected. The material and operational costs as well as the apparatus complexity of the power unit are dependent on the choice of the working fluid. For the ongoing development process, the power unit will be calculated for Helium and Air to estimate the development, maintenance and operational costs.

\section{Literature}

[1] Thomaschko L., "Pelet fired turbine and Ökfen", Presentation kick off Meeting "chilled turbines" consortium, January, 2019; Berlin Moabit .

[2] Bošnjaković, Fran; Knoche, Karl-Friedrich, „Technische Thermodynamik“, 1988, Dresden, Steinkopff, pp. 227-230

[3] Tesla Nikola, "Tesla Turbine", Pat.No. U.S. 1061206, 1909

[4] Lisker R., "Two Phase flow in friction turbines ", Dissertation, University of Rome Tor Vergata, April 2017, pp. 95-100 